\title{
Screening for Psychiatric Disorders in Bariatric Surgery Candidates with the German Version of the Patient Health Questionnaire
}

\author{
Ulrich Palm, ${ }^{1}$ Wolfgang E. Thasler, ${ }^{2}$ Peter Rittler, ${ }^{2}$ Ann Natascha Epple, ${ }^{3}$ \\ Martin Lieb, ${ }^{4}$ Rabee Mokhtari-Nejad, ${ }^{1}$ Susanne Rospleszcz, ${ }^{1}$ Larissa de la Fontaine, ${ }^{1}$ \\ Felix M. Segmiller, ${ }^{1}$ and Daniela Eser-Valeri' ${ }^{1}$ \\ ${ }^{1}$ Department of Psychiatry, Psychotherapy and Psychosomatics, Ludwig-Maximilians University Munich, Nußbaumstraße 7, \\ 80336 Munich, Germany \\ ${ }^{2}$ Department of General, Visceral, Transplantation, Vascular, and Thoracic Surgery, Ludwig-Maximilians University Munich, \\ Marchioninistraße 15, 81377 Munich, Germany \\ ${ }^{3}$ KBO Heckscher Clinic for Childhood and Adolescent Psychiatry, Deisenhofener Straße 28, 81539 Munich, Germany \\ ${ }^{4}$ Privatklinik Meiringen, Willigen, 3860 Meiringen, Switzerland
}

Correspondence should be addressed to Ulrich Palm; ulrich.palm@med.uni-muenchen.de

Received 28 January 2014; Revised 10 April 2014; Accepted 10 April 2014; Published 27 April 2014

Academic Editor: Xingguang Luo

Copyright (C) 2014 Ulrich Palm et al. This is an open access article distributed under the Creative Commons Attribution License, which permits unrestricted use, distribution, and reproduction in any medium, provided the original work is properly cited.

Objective. Obesity has been linked to psychiatric disorders in several studies. Prevalence and severity of psychiatric disorders are high in patients undergoing bariatric surgery. Thus, psychiatric assessment of bariatric surgery candidates has become a standard procedure. However, socially desirable responding leads to biased results in self-reported questionnaires. Here, bariatric surgery candidates were screened with the Patient Health Questionnaire (PHQ-D) additionally to the psychiatric examination. Method. 355 bariatric surgery candidates filled in the PHQ-D before the psychiatric examination as a part of the surgery assessment procedure. PHQ-D results were compared to psychiatric diagnoses and body mass index (BMI). Results. Gender ratio, mean BMI, and age were comparable to earlier studies. Depressive and somatization symptoms did not correlate to BMI. However, females showed higher prevalence of psychiatric disorders with elevated syndrome severity in depressive and somatization disorders, as well as more frequent antidepressant intake. Eating disorders and addiction disorders were rarely reported. Conclusion. The findings suggest a socially desirable responding when filling in the PHQ-D before bariatric surgery. The use of the PHQ-D in this patient sample could be augmented by psychometric tests with internal correction and validation scales. Furthermore, psychiatric examination should be separated from the surgery evaluation process.

\section{Introduction}

Obesity has become a relevant socioeconomic and medical problem in developed and new industrialized countries, leading to a $10 \%$ increase of obesity rates per decade in the USA [1] and an even greater increase in European countries $[2,3]$. In people with a body mass index (BMI) ranging from 30 to $35 \mathrm{~kg} / \mathrm{m}^{2}$, expectancy of life declines by $2-4$ years, in people with a BMI ranging from 40 to $45 \mathrm{~kg} / \mathrm{m}^{2}$ even by $8-$ 10 years [4]. Obese persons, especially women, show higher rates of psychiatric disorders, that is, depressive disorders [5], and they have a higher risk of lifetime depression than normal weight controls [6-12]. Furthermore, prevalence of psychiatric disorders is correlated to increasing BMI [13-15]. Morbidly obese patients (obesity $\mathrm{III}^{\circ}$ ) undergoing bariatric surgery show higher prevalence and severity of psychiatric disorders than people with lower degree of obesity $\left(\mathrm{I}-\mathrm{II}^{\circ}\right)$ [16] or normal weight controls [17-23]. With increasing rates of obesity, bariatric surgery has emerged as an important treatment option in severely obese patients as they do not 
sufficiently respond to standard weight loss programs [24, 25]. Psychiatric assessment of bariatric surgery candidates has become a standard procedure before undergoing bariatric surgery, due to high rates of psychiatric comorbidity [26]. However, a tendency to dissimulation has been observed during the assessment process for bariatric surgery $[12,27$, 28]. Depressive symptoms among bariatric surgery candidates are higher when they are assured that their diagnostic assessment is independent from the suitability rating before bariatric surgery, suggesting that rates of depression are much higher than reported in the respective literature [26, 2931]. As the preoperative diagnosis of depressive or anxiety disorders as well as binge eating disorder is associated with lower weight loss after surgery [32-36], concomitant screening for depressive and other psychiatric disorders during assessment process and follow-up phases has been fostered by additional use of self-reported symptom screening questionnaires, for example, Beck Depression Inventory [23, 37-39]. The PHQ (Patient Health Questionnaire) has been investigated in bariatric surgery candidates in only one study so far. Cassin et al. suggested an elevated cutoff for depression rating in bariatric surgery candidates with the PHQ-9 (module for depressive disorders) due to the interference with somatization symptoms, leading to a false positive result of depressive symptoms [40], and they found a lower sensitivity and specificity of each 0.75 compared to the earlier PHQ studies, suggesting socially desirable responding by the candidates. Here, we aimed at evaluating psychiatric symptoms in a sample of German bariatric surgery candidates with the full German version of the PHQ (PHQ-D) for several reasons: the full PHQ version delivers a clearer discrimination between depressive and somatoform disorders due to the items assessed in the somatoform disorder category. It provides information on additional symptoms relevant for the evaluation process of suitability, for example, eating disorders, alcoholism, stress symptoms, and medication intake. These additional factors are relevant for weighing up absolute/relative contraindications and for screening for socially desirable responding. To our knowledge, the PHQ-D has not been applied in bariatric surgery candidates yet.

\section{Materials and Methods}

2.1. Subjects. All participants were bariatric surgery candidates from the Department of Surgery of the LudwigMaximilians University. Before undergoing surgery, candidates were examined by the Psychiatric Liaison Service of the Department of Psychiatry and gastroenterologic as well as cardiologic evaluation for their suitability. The criteria for considering bariatric surgery were BMI $\geq 40 \mathrm{~kg} / \mathrm{m}^{2}$ or a $\mathrm{BMI} \geq 35 \mathrm{~km} / \mathrm{m}^{2}$ with at least one comorbidity, for example, diabetes mellitus II, hyperlipidemia, sleep apnea, and orthopedic problems [41]. Before the psychiatric examination, subjects were asked to fill in the PHQ-D questionnaire as an additional examination to the standard assessment procedure. They were assured that refusal would not have consequences in terms of the psychiatric assessment and that the collected data were anonymized for scientific use. However, subjects were not informed in advance that the PHQ$\mathrm{D}$ had no impact to the psychiatric assessment procedure. Between 2006 and 2011, more than 700 bariatric surgery candidates were evaluated and a total of 355 patients agreed to fill in the questionnaire.

2.2. $P H Q-D$. The PHQ-D is the German translation of the PRIME-MD Patient Health Questionnaire [42]. The questionnaire has been validated in several studies and showed sufficient validity, sensitivity, specificity, and internal consistency (Cronbach's $\alpha$ for depression scale: 0.88 ; for somatization scale: 0.79) [43-45]. The PHQ-D covers somatization disorders (q. 1), depressive disorders (q. 2), anxiety disorders (q. 3-5), eating disorders (q. 6-8), alcohol abuse (q. 9-10), psychosocial functioning (q. 11), stress symptoms (q. 12), posttraumatic stress disorder (q. 13), intake of psychopharmacologic drugs (q. 15), and questions on premenstrual dysphoric disorder (q. 16). In this study, depressive, somatization, and stress symptoms are calculated by a sum score; all other items are calculated by a cut-off/threshold or a dichotomous criterion (yes/no). Additionally, depressive symptoms are clustered in groups of severity according to the PHQ-D manual [46]. Free answers (q. 14) and the question on psychosocial functioning (q. 11) were excluded for lacking reliability. Premenstrual dysphoric syndrome was assessed dichotomously by q. 16 b.

2.3. Psychiatric Assessment. After filling in the questionnaire, patients underwent a standardized psychiatric examination by an experienced psychiatrist. The semistructured interview included a psychopathological examination, medical and psychiatric history, history of weight gain, eating and drinking characteristics, calculation of actual BMI, former pharmacological and psychotherapeutic treatments, and sociodemographic and economic properties. Additionally, compliance, resilience, and motivational factors for bariatric surgery as well as subjective risk estimation were assessed. If applicable, PHQ-D results were discussed with the patients and they were told that the PHQ-D had no impact on the recommendation or rejection of bariatric surgery. Finally, up to three psychiatric diagnoses were made according to ICD-10 criteria and diagnoses were sorted by their relevance. If there was no relevant psychopathology, no psychiatric diagnosis was made. In addition, obesity was assessed by the ICD-10 categories (degrees I-III). Recommendation for or rejection of bariatric surgery was reported to the department of surgery and the patients.

2.4. Statistical Analyses. Demographic data were measured as mean and standard deviation, where applicable, or median and interquartile range. Incomplete data sets $(n=13)$ or values exceeding two standard deviations were not excluded from analysis to reflect the whole spectrum of results. A level of significance $\leq 0.05$ was accepted. Fisher's exact test was used for detecting gender differences in the PHQ-D categories. Pearson's correlation coefficient was used for correlations between BMI and age, BMI and somatization scale, BMI and 
stress symptoms, stress symptoms and somatization, BMI and depression score, and depression score and stress symptoms. ANOVA was applied to assess the correlation between BMI and depression categories of the PHQ-D, the BMI and the psychiatric diagnoses, and stress symptoms and depression categories. Unadjusted $t$-tests were used for BMI differences in the groups with and without binge eating disorder or bulimia, depression score and antidepressant intake, somatization score and antidepressant intake, and depression score and premenstrual syndrome. The correlation between depression category and antidepressant intake was assessed by a chi-square test. Bonferroni correction was applied where necessary. The statistical program R 2.15.2 (Institute for Statistics and Mathematics, Vienna, Austria) was used for all statistical analyses.

\section{Results}

3.1. Demographic Data and Psychiatric Diagnoses. Overall, 355 bariatric surgery candidates completed the PHQ-D; 271 (76\%) were female. Mean age was $41.8 \pm 12.3$ years with a range of $17-72$ years. Mean BMI was $47.8 \pm 8.4 \mathrm{~kg} / \mathrm{m}^{2}$ with a range of $27-80 \mathrm{~kg} / \mathrm{m}^{2}$. Mean age of female candidates was 41.2 years compared to 43.7 years in male candidates $(P=0.2)$. BMI in males was significantly higher $\left(50.2 \pm 11.3 \mathrm{~kg} / \mathrm{m}^{2}\right)$ than in females $\left(47.0 \pm 9.0 \mathrm{~kg} / \mathrm{m}^{2}\right)(P=0.0054) .190(71 \%)$ female candidates showed a premenstrual syndrome due to PHQ. Obesity categories showed a high prevalence of BMI $\geq$ $40 \mathrm{~kg} / \mathrm{m}^{2}$ (morbid obesity, ICD-10: E66.02) in 309 candidates (87\%), followed by BMI $35-39.9 \mathrm{~kg} / \mathrm{m}^{2}$ (ICD-10: E66.01) in 33 candidates (9\%) and BMI 30-34.9 kg/m² (ICD-10: E66.00) in 11 candidates (3\%). Two male patients had a Prader-Willi syndrome (ICD-10: Q87.1).

Psychiatric diagnoses were clustered to depressive disorders including (recurrent) major depressive disorder and adjustment disorder (ICD-10: F32.x, F33.x, F43.2), eating disorders including bulimia, binge eating disorder, and other eating disorders (ICD: F50.2, F50.4, F50.9), and other psychiatric disorders including addiction disorders, personality disorders, delusional disorders, anxiety disorders, obsessivecompulsive disorders, and posttraumatic stress disorders (ICD-10: F1x.x, F2x.x, F40.x, F41.x, F42.x, F43.1, F60.x). 127 candidates (36\%) had no psychiatric diagnosis at all, 228 subjects (64\%) had at least one psychiatric diagnosis, 23 candidates had a second psychiatric diagnosis, and 4 candidates had a third psychiatric diagnosis. At the first psychiatric diagnosis 161 subjects (70\%) showed a depressive disorder, 44 subjects (20\%) were diagnosed with an eating disorder, and 23 subjects $(10 \%)$ had other psychiatric diagnoses. The second psychiatric diagnosis showed eating disorders in 10 patients (43\%) and other psychiatric disorders in 13 patients $(57 \%)$. The third psychiatric diagnosis consisted in other disorders $(n=4)$. Overall, 161 subjects had a depressive disorder as primary diagnosis; 54 showed an eating disorder and 40 had other psychiatric disorders as primary or concomitant diagnoses. Women had significantly more diagnoses of depressive disorders $(48 \%)$ than men $(36 \%)(P=0.011)$. Further details are reported in Table 1.
3.2. PHQ-D Results. For the whole sample, PHQ-D depression categories showed no depressive symptoms in 104 candidates (29\%), mild depressive symptoms in 119 candidates (34\%), moderate depressive symptoms in 86 candidates (24\%), moderate-severe depressive symptoms in 32 candidates (9\%), and severe depressive symptoms in 13 candidates (4\%). Panic disorder occurred in 23 patients (6\%) and anxiety disorder occurred in 25 subjects (7\%). 26 patients $(7 \%)$ reported bulimia symptoms and 25 patients $(7 \%)$ reached the cut-off for binge eating disorder. Alcohol abuse was reported by only 11 subjects (3\%). PTSD symptoms occurred in 10 patients (3\%). 56 candidates (16\%) reported use of psychopharmacologic medication. Median somatization score was $11 \pm 9$ points (range $0-28$ ) and median stress score was $7 \pm 6$ points (range $0-24$ ).

In male candidates, the category "no depression" was significantly more frequent (39\%) than in female subjects (26\%) $(P=0.046)$ and men showed less severe depressive syndromes $(0 \%)$ than women $(5 \%)$. The somatization scale was significantly increased in female subjects (median $12 \pm 8$; range $0-28)$ compared to male subjects ( \pm \pm 9 ; range $0-$ 23) $(P=0.0005)$ and females showed a significantly more frequent antidepressant intake $(18 \%)$ than males $(7 \%)(P=$ 0.015). All other PHQ-D measures did not show significant gender differences (Table 1).

3.3. Correlation Analyses. Pearson's correlation coefficient showed no significance for BMI and age, BMI and somatization, BMI and stress symptoms, BMI and score of depressive symptoms, BMI and depression categories, BMI and psychiatric diagnoses, BMI and binge eating disorder, and BMI and bulimia. However, women showed a higher (but not significant) correlation coefficient between BMI and clinical relevant depression score (threshold disorder; 5-29 points) than the whole sample $(r=0.099 ; P=0.174)$. Furthermore, in women, BMI increased corresponding to the depression categories "mild," "moderate," and "moderatesevere" ( $P=0.4)$, but not to the category "severe."

Correlation between stress symptoms and somatization was statistically significant $(r=0.592 ; P<0.01)$; also stress symptoms and score of depressive symptoms showed a highly significant correlation $(r=0.667 ; P<0.001)$. Score of stress symptoms was significantly correlated with the depression categories $(P<0.001)$. Depressive symptom scores were associated with medication intake $(P<0.001)$ and showed a mean score of 7.6 in the drug-naive group compared to a mean score of 11.8 in the medicated group. Furthermore, depression categories and antidepressant intake were correlated $(P<0.001)$. Somatization scores were associated with antidepressant intake $(P<0.001)$ and showed a mean score of 10.6 in the drug-naive group compared to a mean score of 14.1 in the medicated group.

\section{Discussion}

In this trial, the German version of the Patient Health Questionnaire was used for screening for psychiatric disorders in a sample of 355 bariatric surgery candidates. 
TABLE 1: Demographic and clinical characteristics.

\begin{tabular}{|c|c|c|c|c|}
\hline & Whole sample & Male & Female & $P$ value \\
\hline$N$ & 355 & $84(24 \%)$ & $271(76 \%)$ & \\
\hline Age (years) & $41.8 \pm 12.3$ & $43.7 \pm 23.5$ & $41.2 \pm 17.0$ & $P=0.2$ \\
\hline BMI $\left(\mathrm{kg} / \mathrm{m}^{2}\right)$ & $47.8 \pm 8.4$ & $50.2 \pm 11.3$ & $47.0 \pm 9.0$ & $P=0.0054^{*}$ \\
\hline \multicolumn{5}{|l|}{ Somatic ICD-10 diagnoses } \\
\hline E66.00 & $11(3 \%)$ & $1(1 \%)$ & $10(4 \%)$ & \multirow{4}{*}{$P=0.11^{\mathrm{a}}$} \\
\hline E66.01 & $33(9 \%)$ & $6(7 \%)$ & $27(10 \%)$ & \\
\hline E66.02 & $309(88 \%)$ & $75(90 \%)$ & $234(86 \%)$ & \\
\hline Q87.1 & $2(1 \%)$ & $2(2 \%)$ & $0(0 \%)$ & \\
\hline \multicolumn{5}{|l|}{ Psychiatric disorders } \\
\hline Depressive disorders & $161(54 \%)$ & $30(36 \%)$ & $131(48 \%)$ & \multirow{4}{*}{$P=0.011^{* a}$} \\
\hline Eating disorders & $44(12 \%)$ & $8(10 \%)$ & $36(13 \%)$ & \\
\hline Other disorders & $23(6 \%)$ & $3(4 \%)$ & $20(7 \%)$ & \\
\hline No disorder & $127(36 \%)$ & $43(51 \%)$ & $84(31 \%)$ & \\
\hline \multicolumn{5}{|l|}{ PHQ depression category } \\
\hline No depression & $105(29 \%)$ & $33(39 \%)$ & $72(26 \%)$ & \multirow{5}{*}{$P=0.046^{* a}$} \\
\hline Mild depression & $119(34 \%)$ & $24(29 \%)$ & $95(35 \%)$ & \\
\hline Moderate depression & $86(24 \%)$ & $18(21 \%)$ & $68(25 \%)$ & \\
\hline Moderate-severe depression & $32(9 \%)$ & $9(11 \%)$ & $23(9 \%)$ & \\
\hline Severe depression & $13(4 \%)$ & $0(0 \%)$ & $13(5 \%)$ & \\
\hline \multicolumn{5}{|l|}{ PHQ other categories } \\
\hline Somatization & $11.2 \pm 9.0$ & $9.3 \pm 7.5$ & $11.8 \pm 8.0$ & $P=0.0005^{*}$ \\
\hline Stress symptoms & $7.8 \pm 6.0$ & $7.0 \pm 6.0$ & $8.1 \pm 6.0$ & $P=0.063$ \\
\hline Panic & $23(6 \%)$ & $5(6 \%)$ & $18(7 \%)$ & $P=1$ \\
\hline Anxiety & $25(7 \%)$ & $5(6 \%)$ & $20(7 \%)$ & $P=0.81$ \\
\hline Bulimia & $26(7 \%)$ & $4(5 \%)$ & $22(8 \%)$ & $P=0.47$ \\
\hline Binge eating & $25(7 \%)$ & $3(4 \%)$ & $22(8 \%)$ & $P=0.22$ \\
\hline Alcoholism & $11(3 \%)$ & $6(7 \%)$ & $5(2 \%)$ & $P=0.025^{*}$ \\
\hline PTSD & $10(3 \%)$ & $1(1 \%)$ & $9(3 \%)$ & $P=0.46$ \\
\hline Medication & $56(16 \%)$ & $6(7 \%)$ & $50(18 \%)$ & $P=0.015^{*}$ \\
\hline
\end{tabular}

${ }^{*}$ Significance $\leq 0.05$.

${ }^{a}$ Significant difference between male and female patients in at least one diagnostic category.

The sociodemographic results of our study are in line with the findings in earlier trials, that is, similar percentage of women (3/4 of the study population), median BMI and age [37, 40, 47, 48]. Krukowski et al. [37] found a similar BMI $\left(49 \mathrm{~kg} / \mathrm{m}^{2}\right)$ in predominantly female patients $(84 \%)$ with a mean age of 43 years, but higher rates of minimal and mild depression in their investigation on the utility of Beck's Depression Inventory in bariatric patients. Cassin et al. [40] found similar BMI $\left(48 \mathrm{~kg} / \mathrm{m}^{2}\right)$ and a mean age (44 years), as well as a high proportion of female gender (77\%). The elevated proportion of female gender, associated with higher depression severity, has been shown in various studies [37, $40,49,50]$ and could be confirmed in our analysis. However, BMI was not correlated to depression severity or somatization in our study. This correlation has been found in most of the studies, and also a negative result has been reported [47]. The prevalence of at least one psychiatric diagnosis in bariatric surgery patients is $64 \%$ in our study and comparable to the respective literature [12]. The prevalence of a second or third psychiatric diagnosis in our sample is lower than in other studies $[6,8-12,18,51]$. Furthermore the low rate of eating disorders, addiction disorders, and medication intake in our sample is in contrast to other studies [52-54]. This discrepancy could point to a strict preselection of bariatric surgery candidates before psychiatric assessment. However, socially desirable responding which has also been reported in other studies $[27,28,48]$ cannot be fully excluded.

The lacking correlation of depression severity and BMI could be not only due to distortion in responding, but also due to the treatment of severe depressive disorders with psychopharmacologic medication, especially in women. This group showed more drug intake than male candidates, higher severity of depressive and somatization symptoms, and increasing depression severity with higher BMI, except for the category of severe depression. Although this correlation did not reach significance, it could be speculated that females with severe depression are more likely medicated with antidepressants, masking a correlation between depression and BMI that probably could be seen if there was no antidepressant intake. The illness severity may be 
underlined by the elevated score of depressive and somatization symptoms which is higher despite drug intake compared to patients without medication. Overall, the medicated group presented a median depression score of 11.8 points (moderate depression), indicating a potentially higher depression severity without antidepressant intake.

The significant correlations between somatization $\times$ stress, depression score $\times$ stress, and depressive, respectively, somatization symptoms $\times$ medication intake confirms the good internal consistency of the questionnaire and shows a higher prevalence and severity of psychiatric syndromes in female candidates despite having lower BMI than males. Females with premenstrual dysphoric syndrome show higher depression score than females without, and females are more likely to be medicated with psychotropic drugs.

4.1. Limitations. The lack of a correlation between BMI and psychopathologic symptoms in our study is rather surprising in regard to several studies showing clear correlation between BMI and psychiatric disorders. Thus, elevated BMI is supposed to provoke more physical restraints and disabilities, leading to increased somatization symptoms. Social stigmatization following elevated BMI could also be responsible for depressive feelings. However, there are some studies without a clear correlation between BMI and psychiatric disorders, pointing to a socially desirable responding in bariatric surgery candidates. It is possible that socially desirable responding in the self-administered PHQ could have led to our results. This bias in responding is probably most prominent in the categories of eating disorders and alcohol syndrome, where the prevalence is quite lower than in the respective literature, and also the categories with stress-related questions and somatization/depression could be biased. Another proposed explanation for potential bias is the diagnostic process made by the psychiatrist, bearing in mind that anamnesis underlies subjective statements and the psychiatric assessment process is probably too short to investigate discrepancies [12]. Although our evaluation process was standardized and executed by experienced psychiatrists, this potential bias cannot be fully ruled out. For this reason the use of psychometric rating instruments with internal correction and validity scales like the Minnesota Multiphasic Personality Inventory-2 (MMPI-2) has been suggested [55]. Furthermore the PHQ did not reach the same diagnostic sensitivity compared to other psychometric tests [56, 57], especially regarding dysthymia [57]. This could lead to a distortion of the prevalence of depressive disorders.

Other more psychodynamic mechanisms could involve an unconscious neglect of physical and psychological burden or an altered subjective cause-effect relationship without mandatory conjunction of weight and burden. This could be an explanation for the finding that somatization/depression score and psychosocial stress showed significant correlation, whereas physical burden (expressed by BMI) and psychological burden showed no correlation. Additionally, socioeconomic and culture-bound heterogeneity of the sample could have influenced subjective rating of obesity and psychological burden [58]. Another possible explanation could be an adaptation process of obese patients, leading to neutral subjective rating of objectively severe restraints and impairments [49].

4.2. Implications for the Use of the PHQ. The PHQ-D can easily be used as a screening tool for psychiatric disorders in bariatric surgery candidates. The questionnaire is comprehensible for patients, the summary is made quickly, and the results can be used for further distinct questions on abnormalities. However, bearing in mind the lack of sensitivity in the depression/dysthymia domain and the problem of socially desirable responding, the use of the PHQ as an indicator for the direction of the psychiatric assessment is by no means sufficient. Contrarily, the psychiatric interview should cover all relevant topics at first; afterwards the PHQ could be used to screen for additional symptoms or to discuss discrepancies between the psychiatric assessment and the PHQ result. Due to the lack of internal correction and validation scales in the PHQ, additional psychometric tests could be used. Recent investigations showed that even standardized assessments (e.g., Structured Clinical Interview for DSM-IV - SCID) had low concordance with diagnoses made by subjective psychiatric exploration $[59,60]$. Thus, a standardized assessment process with reliable diagnostic tools has been recommended, leading to reduced bias by both patients and psychiatrists and yielding sufficient discriminatory power between the different syndromes. Additionally, the evaluation process for bariatric surgery could be separated from the psychiatric interview in order to achieve less bias in reporting severe symptoms [61]. If the fear of being rejected from bariatric surgery could be reduced by a separated evaluation process, the bias in self-reported symptom severity could decline as well. In this case, the PHQ(-D) might be useful as a screening instrument before and in the follow-up of bariatric surgery.

\section{Conclusion}

The PHQ-D has been investigated to screen for psychiatric disorders in a large sample of bariatric surgery candidates. Depression, somatization severity, and medication intake were more frequent in female than in male patients. However, BMI was not correlated to depression and somatization score. This could be due to biases in self-reporting psychiatric symptoms, a also due to a lack of concordance in psychiatric diagnoses and test results. The lack of correlation between PHQ categories and BMI hampers the use of the PHQ as a standard or first line assessment tool in bariatric surgery candidates. Thus, psychiatric evaluation should rely on the diagnostic interview and psychometric tests with internal correction and validation scales. A future standardized procedure for the assessment of bariatric surgery candidates could split the evaluation rating from the psychiatric diagnosis, leading to the reduction of bias in self-reporting due to the fear of rejection from surgery. In this case, the PHQ could be used to screen before and after bariatric surgery for depicting the course of psychiatric disorders. However, further studies are needed to elucidate the use of the PHQ(-D) in bariatric surgery candidates. 


\section{Conflict of Interests}

The authors declare that there is no conflict of interests.

\section{Acknowledgments}

This work is part of the M.D. degree thesis of Ann Natascha Epple. The authors thank Angela Poeller for data collection.

\section{References}

[1] K. M. Flegal, M. D. Carroll, C. L. Ogden, and C. L. Johnson, "Prevalence and trends in obesity among US adults, 1999-2000," Journal of the American Medical Association, vol. 288, no. 14, pp. 1723-1727, 2002.

[2] R. Hyde, "Europe battles with obesity," The Lancet, vol. 371, no. 9631, pp. 2160-2161, 2008.

[3] G. B. M. Mensink, T. Lampert, and E. Bergmann, "Overweight and obesity in Germany 1984-2003," BundesgesundheitsblattGesundheitsforschung-Gesundheitsschutz, vol. 48, no. 12, pp. 1348-1356, 2005.

[4] K. R. Fontaine, D. T. Redden, C. Wang, A. O. Westfall, and D. B. Allison, "Years of life lost due to obesity," Journal of the American Medical Association, vol. 289, no. 2, pp. 187-193, 2003.

[5] L. De Wit, F. Luppino, A. van Straten, B. Penninx, F. Zitman, and P. Cuijpers, "Depression and obesity: a meta-analysis of community-based studies," Psychiatry Research, vol. 178, no. 2, pp. 230-235, 2010.

[6] K. A. Halmi, M. Long, A. J. Stunkard, and E. Mason, "Psychiatric diagnosis of morbidly obese gastric bypass patients," American Journal of Psychiatry, vol. 137, no. 4, pp. 470-472, 1980.

[7] M. Q. Werrij, S. Mulkens, H. J. Hospers, and A. Jansen, "Overweight and obesity: the significance of a depressed mood," Patient Education and Counseling, vol. 62, no. 1, pp. 126-131, 2006.

[8] R. Gertler and G. Ramsey-Stewart, "Pre-operative psychiatric assessment of patients presenting for gastric bariatric surgery (surgical control of morbid obesity)," Australian and New Zealand Journal of Surgery, vol. 56, no. 2, pp. 157-161, 1986.

[9] J. Glinski, S. Wetzler, and E. Goodman, "The psychology of gastric bypass surgery," Obesity Surgery, vol. 11, no. 5, pp. 581588, 2001.

[10] F. Larsen, "Psychosocial function before and after gastric banding surgery for morbid obesity. A prospective psychiatric study," Acta Psychiatrica Scandinavica, Supplement, vol. 82, no. 359, pp. 1-57, 1990.

[11] P. S. Powers, A. Rosemurgy, F. Boyd, and A. Perez, "Outcome of gastric restriction procedures: weight, psychiatric diagnoses, and satisfaction," Obesity Surgery, vol. 7, no. 6, pp. 471-477, 1997.

[12] D. B. Sarwer, N. I. Cohn, L. M. Gibbons et al., "Psychiatric diagnoses and psychiatric treatment among bariatric surgery candidates," Obesity Surgery, vol. 14, no. 9, pp. 1148-1156, 2004.

[13] M. Heo, A. Pietrobelli, K. R. Fontaine, J. A. Sirey, and M. S. Faith, "Depressive mood and obesity in US adults: comparison and moderation by sex, age, and race," International Journal of Obesity, vol. 30, no. 3, pp. 513-519, 2006.

[14] G. E. Simon, M. Von Korff, K. Saunders et al., "Association between obesity and psychiatric disorders in the US adult population," Archives of General Psychiatry, vol. 63, no. 7, pp. 824-830, 2006.
[15] H. Baumeister and M. Härter, "Mental disorders in patients with obesity in comparison with healthy probands," International Journal of Obesity, vol. 6, pp. 1-10, 2007.

[16] C. U. Onyike, R. M. Crum, H. B. Lee, C. G. Lyketsos, and W. W. Eaton, "Is obesity associated with major depression? Results from the third National Health and Nutrition Examination Survey," American Journal of Epidemiology, vol. 158, no. 12, pp. 1139-1147, 2003.

[17] D. W. Black, W. R. Yates, J. H. Reich, S. Bell, R. B. Goldstein, and E. E. Mason, "DSM-III personality disorder in bariatric clinic patients," Annals of Clinical Psychiatry, vol. 1, no. 1, pp. 33-37, 1989.

[18] D. W. Black, R. B. Goldstein, and E. E. Mason, "Prevalence of mental disorder in 88 morbidly obese bariatric clinic patients," American Journal of Psychiatry, vol. 149, no. 2, pp. 227-234, 1992.

[19] S. Herpertz, R. Burgmer, A. Stang et al., "Prevalence of mental disorders in normal-weight and obese individuals with and without weight loss treatment in a German urban population," Journal of Psychosomatic Research, vol. 61, no. 1, pp. 95-103, 2006.

[20] L. Sjostrom, B. Larsson, L. Backman et al., "Swedish obese subjects (SOS). Recruitment for an intervention study and a selected description of the obese state," International Journal of Obesity, vol. 16, no. 6, pp. 465-479, 1992.

[21] M. Sullivan, J. Karlsson, L. Sjöström et al., "Swedish obese subjects (SOS) - an intervention study of obesity. Baseline evaluation of health and psychosocial functioning in the first 1743 subjects examined," International Journal of Obesity and Related Metabolic Disorders, vol. 17, no. 9, pp. 503-512, 1993.

[22] T. A. Wadden, D. B. Sarwer, L. G. Womble, G. D. Foster, B. G. McGuckin, and A. Schimmel, "Psychosocial aspects of obesity and obesity surgery," Surgical Clinics of North America, vol. 81, no. 5, pp. 1001-1024, 2001.

[23] T. A. Wadden, M. L. Butryn, D. B. Sarwer et al., "Comparison of psychosocial status in treatment-seeking women with class III vs. Class I-II obesity," Surgery for Obesity and Related Diseases, vol. 2, no. 2, pp. 138-145, 2006.

[24] C. Ayyad and T. Andersen, "Long-term efficacy of dietary treatment of obesity: a systematic review of studies published between 1931 and 1999," Obesity Reviews, vol. 1, no. 2, pp. 113119,2000

[25] G. K. Goodrick and J. P. Foreyt, "Why treatments for obesity don't last," Journal of the American Dietetic Association, vol. 91, no. 10, pp. 1243-1247, 1991.

[26] M. A. Kalarchian, M. D. Marcus, M. D. Levine et al., "Psychiatric disorders among bariatric surgery candidates: relationship to obesity and functional health status," American Journal of Psychiatry, vol. 164, no. 2, pp. 328-334, 2007.

[27] S. Ambwani, A. G. Boeka, J. D. Brown et al., "Socially desirable responding by bariatric surgery candidates during psychological assessment," Surgery for Obesity and Related Diseases, vol. 9, no. 2, pp. 300-305, 2003.

[28] A. N. Fabricatore, D. B. Sarwer, T. A. Wadden, C. J. Combs, and J. L. Krasucki, "Impression management or real change? Reports of depressive symptoms before and after the preoperative psychological evaluation for bariatric surgery," Obesity Surgery, vol. 17, no. 9, pp. 1213-1219, 2007.

[29] B. Mühlhans, T. Horbach, and M. de Zwaan, "Psychiatric disorders in bariatric surgery candidates: a review of the literature and results of a German prebariatric surgery sample," General Hospital Psychiatry, vol. 31, no. 5, pp. 414-421, 2009. 
[30] M. Mauri, P. Rucci, A. Calderone et al., "Axis I and II disorders and quality of life in bariatric surgery candidates," Journal of Clinical Psychiatry, vol. 69, no. 2, pp. 295-301, 2008.

[31] P. H. Rosenberger, K. E. Henderson, and C. M. Grilo, "Psychiatric disorder comorbidity and association with eating disorders in bariatric surgery patients: a cross-sectional study using structured interview-based diagnosis," Journal of Clinical Psychiatry, vol. 67, no. 7, pp. 1080-1085, 2006.

[32] Y. Averbukh, S. Heshka, H. El-Shoreya et al., "Depression score predicts weight loss following roux-en-Y gastric bypass," Obesity Surgery, vol. 13, no. 6, pp. 833-836, 2003.

[33] P. Brunault, D. Jacobi, V. Miknius et al., "High preoperative depression, phobic anxiety, and binge eatings scores and low medium-term weight loss in sleeve gastrectomy obese patients: a preliminary cohort study," Psychosomatics, vol. 53, pp. 363370, 2012.

[34] M. De Zwaan, J. Enderle, S. Wagner et al., "Anxiety and depression in bariatric surgery patients: a prospective, follow-up study using structured clinical interviews," Journal of Affective Disorders, vol. 133, no. 1-2, pp. 61-68, 2011.

[35] T. Legenbauer, M. de Zwaan, A. Benecke, B. Mühlhans, F. Petrak, and S. Herpertz, "Depression and anxiety: their predictive function for weight loss in obese individuals," Obesity Facts, vol. 2, no. 4, pp. 227-234, 2009.

[36] T. Legenbauer, F. Petrak, M. de Zwaan, and S. Herpertz, "Influence of depressive and eating disorders on short- and longterm course of weight after surgical and nonsurgical weight loss treatment," Comprehensive Psychiatry, vol. 52, no. 3, pp. 301-311, 2011.

[37] R. A. Krukowski, K. E. Friedman, and K. L. Applegate, "The utility of the beck depression inventory in a bariatric surgery population," Obesity Surgery, vol. 20, no. 4, pp. 426-431, 2010.

[38] D. J. Munoz, E. Chen, S. Fischer et al., "Considerations for the use of the beck depression inventory in the assessment of weight-loss surgery seeking patients," Obesity Surgery, vol. 17, no. 8, pp. 1097-1101, 2007.

[39] M. J. Hayden, W. A. Brown, L. Brennan, and O. 'Brien PE, "Validity of the Beck Depression Inventory as a screening tool for a clinical mood disorder in bariatric surgery candidates," Obesity Surgery, vol. 22, pp. 1666-1675, 2012.

[40] S. Cassin, S. Sockalingam, R. Hawa et al., "Psychometric properties of the Patient Health Questionnnaire (PHQ-9) as a depression screening tool for bariatric surgery candidates," Psychosomatics, vol. 54, pp. 352-358, 2013.

[41] NIH (National Institutes of Health) Consensus Statement, "Gastrointestinal surgery for severe obesity," Consens Statement, vol. 279, pp. 1-20, 1991.

[42] R. L. Spitzer, K. Kroenke, and J. B. W. Williams, "Validation and utility of a self-report version of PRIME-MD: The PHQ Primary Care Study," Journal of the American Medical Association, vol. 282, no. 18, pp. 1737-1744, 1999.

[43] K. Gräfe, S. Zipfel, W. Herzog, and B. Löwe, "Screening psychischer Störungen mit dem, Gesundheitsfragebogen für Patienten (PHQ-D)," Ergebnisse der Deutschen Validierungsstudie. Diagnostica, vol. 50, pp. 171-181, 2004.

[44] B. Löwe, K. Gräfe, S. Zipfel, S. Witte, B. Loerch, and W. Herzog, "Diagnosing ICD-10 depressive episodes: superior criterion validity of the Patient Health Questionnaire," Psychotherapy and Psychosomatics, vol. 73, no. 6, pp. 386-390, 2004.
[45] B. Löwe, R. L. Spitzer, K. Gräfe et al., "Comparative validity of three screening questionnaires for DSM-IV depressive Disorders and physician's diagnoses," Journal of Affective Disorders, vol. 78, pp. 131-140, 2004.

[46] B. Löwe, R. L. Spitzer, and W. Herzog, PHQ-D: Gesundheitsfragebogen Für Patienten, Pfizer, Karlsruhe, Germany, 2002.

[47] M. R. Ali, J. J. Rasmussen, J. B. Monash, and W. D. Fuller, "Depression is associated with increased severity of comorbidities in bariatric surgical candidates," Surgery for Obesity and Related Diseases, vol. 5, no. 5, pp. 559-564, 2009.

[48] L. R. Jones-Corneille, T. A. Wadden, D. B. Sarwer et al., "Axis i psychopathology in bariatric surgery candidates with and without binge eating disorder: results of structured clinical interviews," Obesity Surgery, vol. 22, no. 3, pp. 389-397, 2012.

[49] G. M. Papageorgiou, A. Papakonstantinou, E. Mamplekou, I. Terzis, and J. Melissas, "Pre- and postoperative psychological characteristics in morbidly obese patients," Obesity Surgery, vol. 12, no. 4, pp. 534-539, 2002.

[50] R. L. Kolotkin, R. D. Crosby, R. Pendleton, M. Strong, R. E. Gress, and T. Adams, "Health-related quality of life in patients seeking gastric bypass surgery vs non-treatment-seeking controls," Obesity Surgery, vol. 13, no. 3, pp. 371-377, 2003.

[51] H. Ø. Lier, E. Biringer, B. Stubhaug, H. R. Eriksen, and T. Tangen, "Psychiatric disorders and participation in Pre- and postoperative counselling groups in bariatric surgery patients," Obesity Surgery, vol. 21, no. 6, pp. 730-737, 2011.

[52] M. De Zwaan, J. E. Mitchell, L. Michael Howell et al., "Characteristics of morbidly obese patients before gastric bypass surgery," Comprehensive Psychiatry, vol. 44, no. 5, pp. 428-434, 2003.

[53] J. E. Mitchell and M. P. Mussell, "Comorbidity and binge eating disorder," Addictive Behaviors, vol. 20, no. 6, pp. 725-732, 1995.

[54] C. F. Telch and E. Stice, "Psychiatric comorbidity in women with binge eating disorder: prevalence rates from a non-treatmentseeking sample," Journal of Consulting and Clinical Psychology, vol. 66, no. 5, pp. 768-776, 1998.

[55] S. Walfish, D. Vance, and A. N. Fabricatore, "Psychological evaluation of bariatric surgery applicants: procedures and reasons for delay or denial of surgery," Obesity Surgery, vol. 17, no. 12, pp. 1578-1583, 2007.

[56] K. A. Wittkampf, H. van Ravesteijn, K. D. Baas et al., "The accuracy of Patient Health Questionnaire-9 in detecting depression and measuring depression severity in high-risk groups in primary care," General Hospital Psychiatry, vol. 31, no. 5, pp. 451459, 2009.

[57] S. M. Eack, C. G. Greeno, and B.-J. Lee, "Limitations of the patient health questionnaire in identifying anxiety and depression in community mental health: many cases are undetected," Research on Social Work Practice, vol. 16, no. 6, pp. 625-631, 2006.

[58] J. F. Kinzl, C. Maier, and A. Bösch, "Morbidly obese patients: psychopathology and eating disorders-results of a preoperative evaluation," Neuropsychiatr, vol. 26, pp. 159-165, 2012.

[59] A. Schlick, S. A. Wagner, B. Mühlhans et al., "Agreement between clinical evaluation and structured clinical interviews (SCID for DSM-IV) in morbidly obese pre-bariatric surgery patients," Psychotherapie Psychosomatik Medizinische Psychologie, vol. 4, pp. 640-646, 2010.

[60] J. E. Mitchell, K. J. Steffen, M. de Zwaan, T. W. Ertelt, J. M. Marino, and A. Mueller, "Congruence between clinical and research-based psychiatric assessment in bariatric surgical 
candidates," Surgery for Obesity and Related Diseases, vol. 6, no. 6, pp. 628-634, 2010.

[61] R. I. Berkowitz and A. N. Fabricatore, "Obesity, psychiatric status, and psychiatric medications," Psychiatric Clinics of North America, vol. 34, no. 4, pp. 747-764, 2011. 


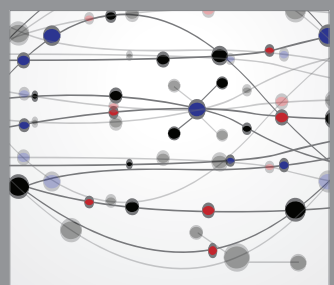

The Scientific World Journal
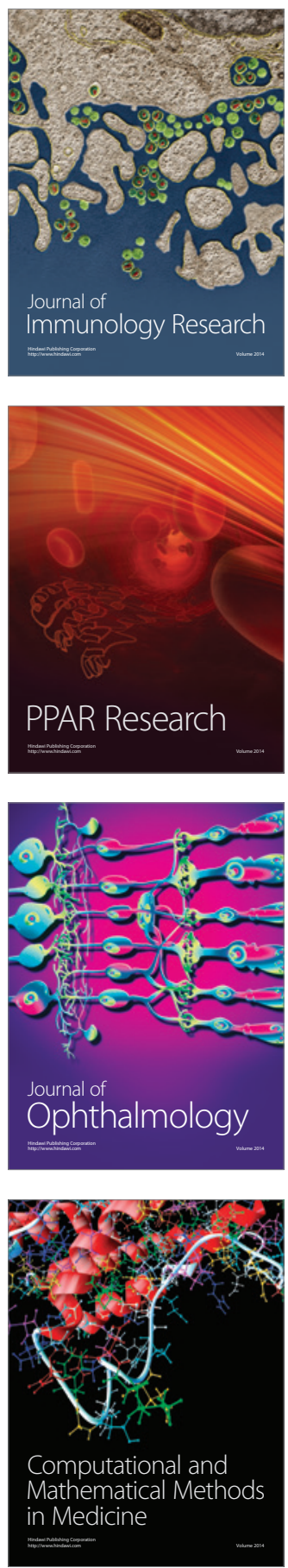

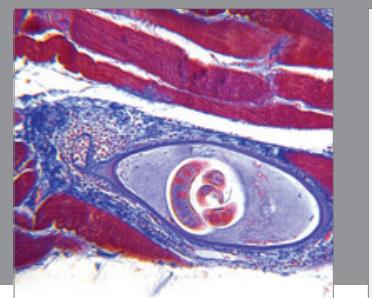

Gastroenterology

Research and Practice
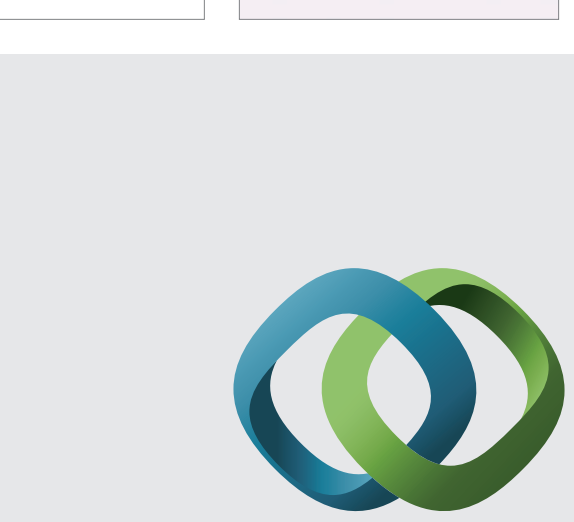

\section{Hindawi}

Submit your manuscripts at

http://www.hindawi.com
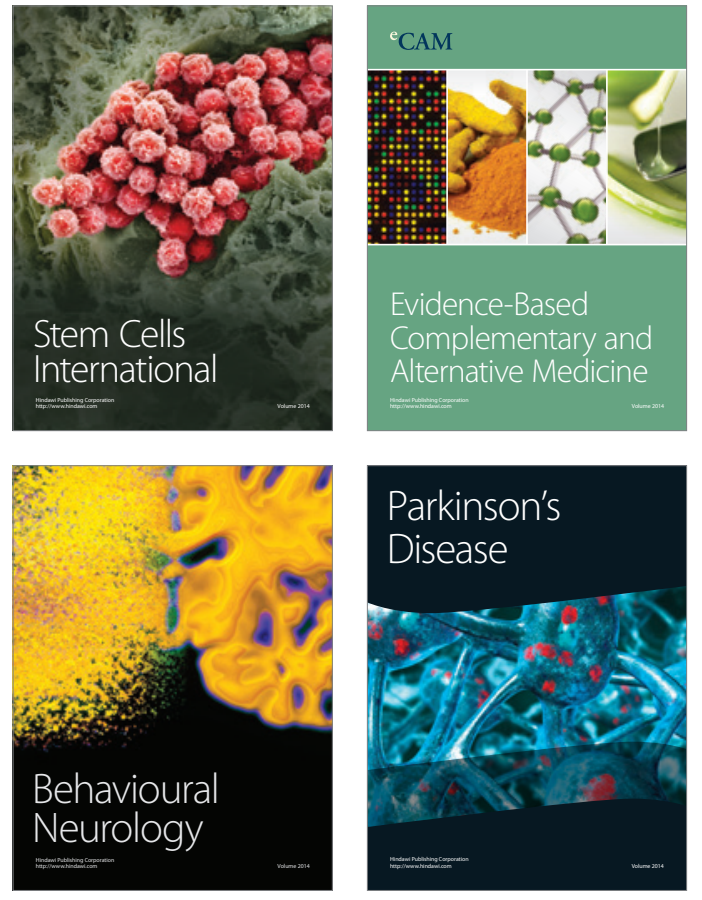
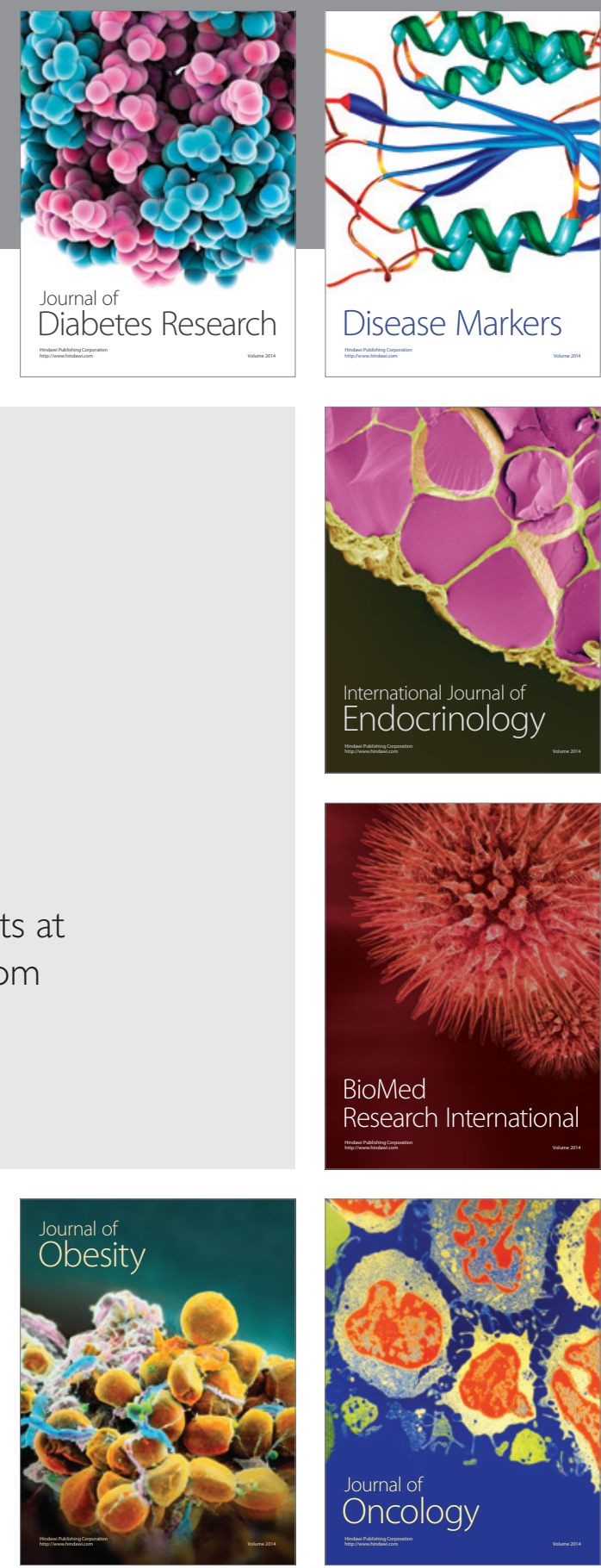

Disease Markers
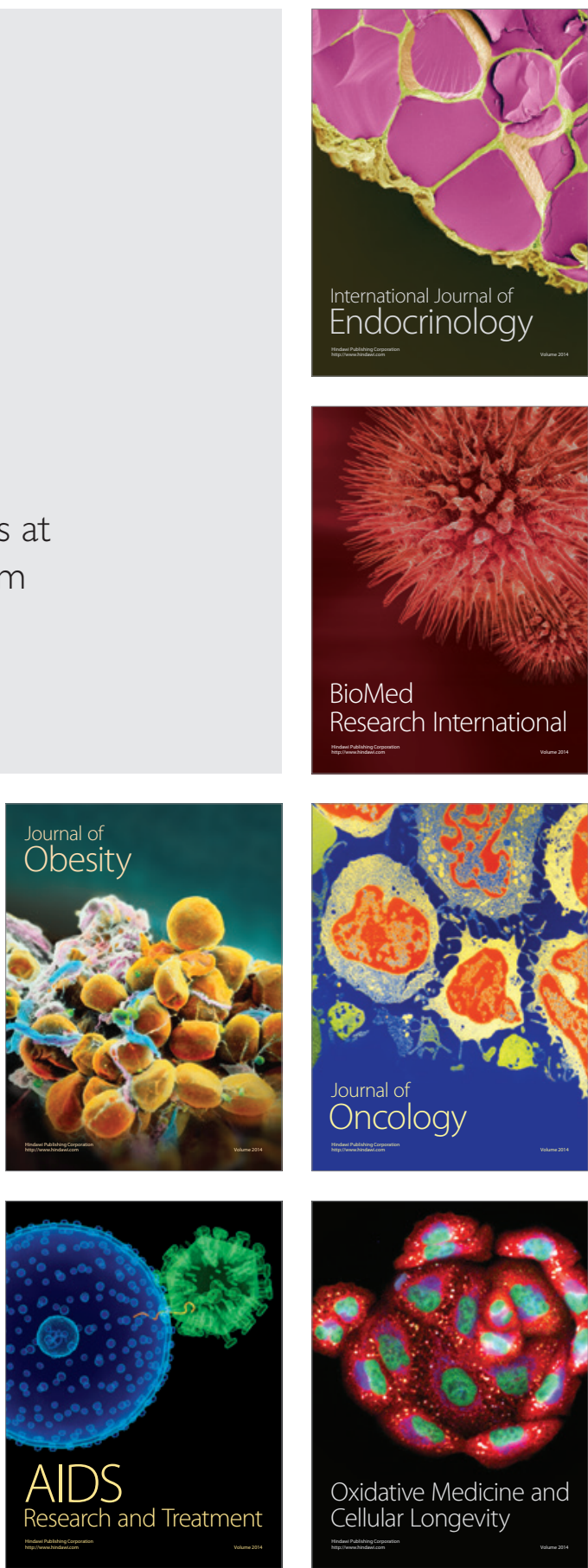